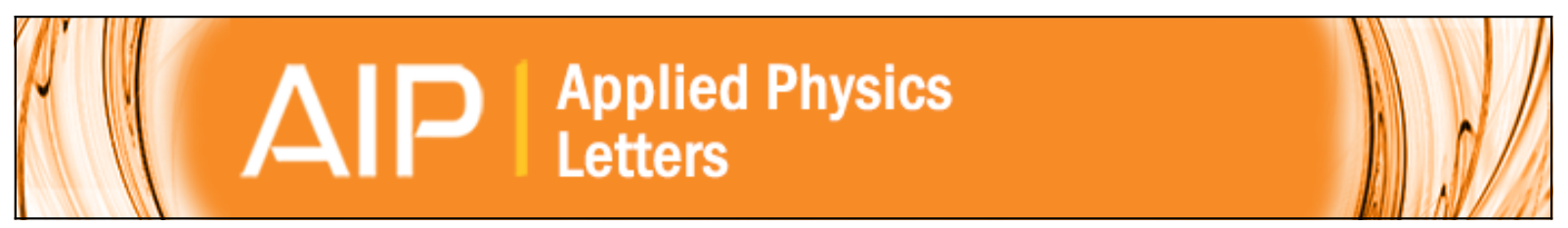

\title{
Nonlinear motion and mechanical mixing in as-grown GaAs nanowires
}

F. R. Braakman, D. Cadeddu, G. Tütüncüoglu, F. Matteini, D. Rüffer, A. Fontcuberta i Morral, and M. Poggio

Citation: Applied Physics Letters 105, 173111 (2014); doi: 10.1063/1.4900928

View online: http://dx.doi.org/10.1063/1.4900928

View Table of Contents: http://scitation.aip.org/content/aip/journal/apl/105/17?ver=pdfcov

Published by the AIP Publishing

\section{Articles you may be interested in}

Surface optical phonons in GaAs nanowires grown by Ga-assisted chemical beam epitaxy

J. Appl. Phys. 115, 034307 (2014); 10.1063/1.4862742

Structural and optical characterization of Mg-doped GaAs nanowires grown on GaAs and Si substrates

J. Appl. Phys. 114, 183508 (2013); 10.1063/1.4829455

GaAs nanowires grown on Al-doped $\mathrm{ZnO}$ buffer layer

J. Appl. Phys. 114, 084309 (2013); 10.1063/1.4819797

Facet structure of GaAs nanowires grown by molecular beam epitaxy

Appl. Phys. Lett. 91, 083106 (2007); 10.1063/1.2769401

GaAs nanowires on Si substrates grown by a solid source molecular beam epitaxy

Appl. Phys. Lett. 89, 053106 (2006); 10.1063/1.2245348

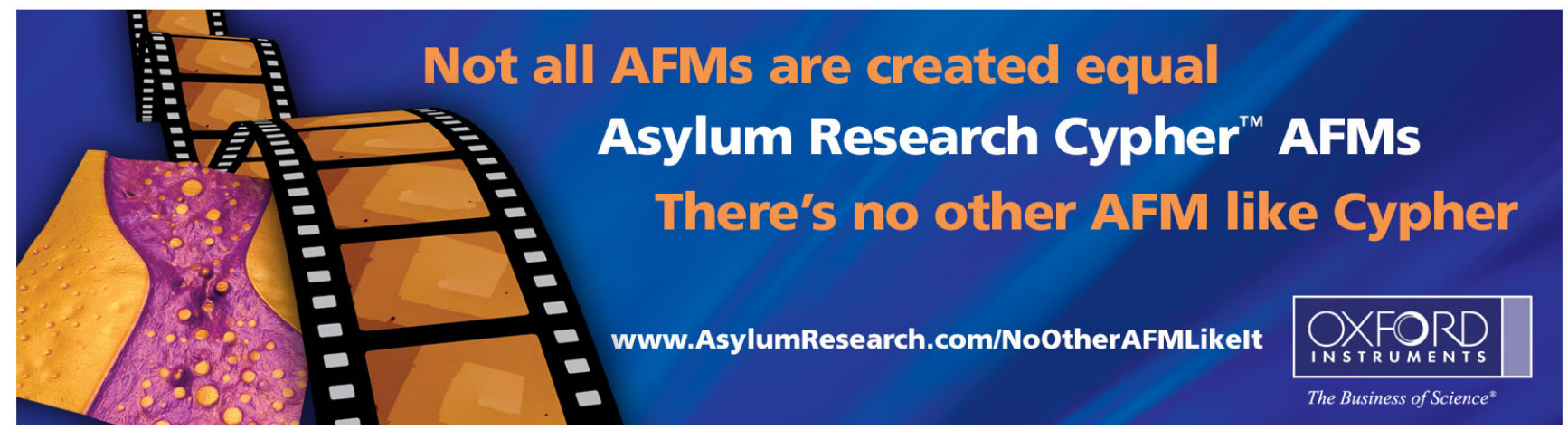




\title{
Nonlinear motion and mechanical mixing in as-grown GaAs nanowires
}

\author{
F. R. Braakman, ${ }^{1, a)}$ D. Cadeddu, ${ }^{1}$ G. Tütüncüoglu, ${ }^{2}$ F. Matteini, ${ }^{2}$ D. Rüffer, ${ }^{2}$ \\ A. Fontcuberta i Morral, ${ }^{2}$ and M. Poggio ${ }^{1}$ \\ ${ }^{1}$ University of Basel, Klingelbergstrasse 82, 4056 Basel, Switzerland \\ ${ }^{2}$ École Polytechnique Fédérale de Lausanne, 1015 Lausanne, Switzerland
}

(Received 23 June 2014; accepted 19 October 2014; published online 30 October 2014)

\begin{abstract}
We report nonlinear behavior in the motion of driven nanowire cantilevers. The nonlinearity can be described by the Duffing equation and is used to demonstrate mechanical mixing of two distinct excitation frequencies. Furthermore, we demonstrate that the nonlinearity can be used to amplify a signal at a frequency close to the mechanical resonance of the nanowire oscillator. $\mathrm{Up}$ to $26 \mathrm{~dB}$ of amplitude gain is demonstrated in this way. (C) 2014 AIP Publishing LLC.
\end{abstract}

[http://dx.doi.org/10.1063/1.4900928]

Due to their favorable geometry and potentially defectfree growth, nanowire cantilevers are promising as ultrasensitive force transducers for scanning probe microscopy. ${ }^{1-4}$ Additionally, their relatively high mechanical resonance frequencies decouple their motion to a large degree from external noise sources and should permit improved sensitivity in mass-sensing and scanning probe applications. Furthermore, the wide choice of nanowire growth material and the possibility to grow nanowire heterostructures could allow access to different measurement modalities, such as sensing of local electric or magnetic fields. In recent experiments, ${ }^{5,6}$ coupling of optical transitions of a self-assembled quantum dot embedded in a nanowire to the motion of the nanowire through strain was demonstrated, opening the way to investigation of hybrid devices with nanowires as their main building blocks. Nanowire heterostructures are attractive as hybrid systems, as they can combine multiple functionalities in one integrated structure.

Conventionally, in scanning probe experiments, oscillatory motion of the cantilever is driven with amplitudes small enough to remain in the linear dynamical regime. Due to a number of reasons, ${ }^{7,8}$ including the oscillator geometry, nonlinear damping, ${ }^{9,10}$ the presence of external potentials, and nonlinear boundary conditions, ${ }^{11,12}$ this linear dynamic range is often quite limited in nanoscale oscillators. ${ }^{13-15}$ The nonlinear dynamics occurring when this range is exceeded complicate the analysis of sensing experiments and are therefore generally avoided or compensated for. ${ }^{16}$ However, nonlinearities in general can also give rise to a host of useful effects, such as signal amplification, ${ }^{17,18}$ noise squeezing, ${ }^{19}$ and frequency mixing. ${ }^{20}$ The nonlinear dynamics of nanowire cantilevers can enable these effects at the nanoscale in mechanical form and have the potential to enhance the performance of cantilever-based sensors.

In this letter, we study the motion of several GaAs nanowires attached to their GaAs growth substrate (Fig. 1(a)). We observe that upon driving the periodic bending motion of a nanowire with sufficiently large amplitudes, it can no longer be described by a linear equation of motion. Instead, the nanowire follows the, qualitatively different, nonlinear

\footnotetext{
${ }^{\text {a) }}$ Author to whom correspondence should be addressed. Electronic mail: floris.braakman@unibas.ch.
}

dynamics of a Duffing oscillator. ${ }^{21}$ A Duffing nonlinearity can give rise to complex motion of an oscillator, such as hysteresis, cascades of period-doubling, and chaotic motion. ${ }^{22}$ In the quantum regime, Duffing nonlinearities have recently been studied in the context of mechanical squeezing. ${ }^{23}$ Furthermore, we find that when applying two driving frequencies, the nanowire motion in the nonlinear regime contains components at frequencies other than the two driving frequencies, as a result of mechanical mixing.

The nanowires under investigation here were grown perpendicularly to their GaAs growth substrate and are still attached to this substrate (see Fig. 1(a)). Using the nanowires as they are grown minimizes the introduction of defects and maintains good clamping of the nanowires to the substrate. Both these factors should decrease damping of the mechanical resonance of the nanowires. The nanowires were grown on a $4 \mathrm{~nm} \mathrm{SiO}_{\mathrm{x}}$ coated (111)B GaAs substrate by the catalyst-free gallium-assisted method ${ }^{24}$ in a DCA P600 solid source molecular beam epitaxy system. Growth has been done under a rotation of $7 \mathrm{rpm}$, with a growth rate of $0.5 \AA / \mathrm{s}$ and a substrate temperature of $630^{\circ} \mathrm{C}$. These GaAs nanowires are of particular interest, since the same structures are straightforwardly coated by subsequent growth steps with a AlGaAs/GaAs shell hosting self-assembled optical quantum dots. $^{25}$

The nanowires mostly exhibit zinc-blende crystal structure and therefore have hexagonal cross-sections, with typical diameters of $100 \mathrm{~nm}$ and lengths up to $25 \mu \mathrm{m}$. The diameter of individual nanowires is constant, except for the tip where a gallium droplet is formed during growth and the base, where small anomalous structures can be present. The nanowires have their fundamental mechanical resonances at frequencies of $f_{0}=1.25-1.35 \mathrm{MHz}$ (some nanowires show two closely spaced resonances, which we attribute to two transverse flexural modes that are non-degenerate due to a slight asymmetry of the nanowire cross-section). Numerical calculations following the same method as used in Ref. 6 confirm that these resonances indeed correspond to the fundamental flexural modes of the singly clamped nanowires.

Furthermore, these resonances exhibit quality factors of up to 37000 (at a temperature of $4.2 \mathrm{~K}$ and pressure below $10^{-6}$ mbar), as determined from the width of the resonance as well as from the ringdown measurements (ringdown 

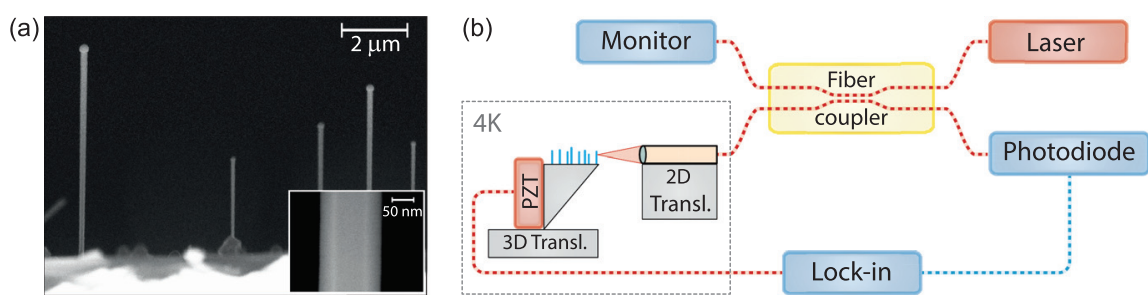

FIG. 1. (a) Scanning electron micrograph of a substrate containing multiple GaAs nanowires, taken at a different section of the same wafer that was used in the experiments. Inset: close-up of a single nanowire, showing a faceted structure due to its hexagonal cross-section. (b) Schematic diagram of the measurement setup.

measurements sometimes show beating patterns, which we again ascribe to the presence of two transverse modes). Many factors contribute to limiting the quality factor of nanomechanical resonators, including lattice defects, which would at this scale likely be dominated by surface defects, surface oxides, clamping losses, and coupling to other mechanical modes. ${ }^{26,27}$ Mechanical damping rates in grown nanowires have been demonstrated to be much lower than for the best cantilevers produced in a top-down manner. ${ }^{2}$ The quality factors observed for the as-grown nanowires studied here are up to an order of magnitude larger than for similar GaAs-based nanowires, ${ }^{5}$ which is a possible indication of the degrading effect of post-processing on the mechanical quality factor.

A schematic overview of the measurement setup is shown in Figure 1(b). The displacement of a nanowire is measured via a fiber-based interferometric method. ${ }^{28}$ In this setup, the nanowire forms one reflecting interface of a lowfinesse Fabry-Pérot interferometer, while the cleaved surface of a single-mode fiber forms the other interface. The sample is mounted on a stack of positioning stages for three-axis translation control, allowing the nanowire of choice to be positioned in the focal plane of an objective placed in front of the single-mode fiber. A voltage-controlled piezoelectric transducer (PZT) attached to the sample holder is used to drive oscillatory bending motion of the nanowires along the optical axis of the interferometer. A fiber coupler is used to inject light from a laser with a wavelength of $1550 \mathrm{~nm}$ into the interferometer. This wavelength is chosen in order to avoid spurious heating of the GaAs nanowires through absorption. The coupler diverts $5 \%$ of the laser power towards the nanowire, resulting in a maximum power incident on the nanowire of $\sim 5 \mu \mathrm{W}$. Varying the laser power slightly did not qualitatively change the measurements, ensuring that no significant heating of the nanowire is taking place. The light reflected by the interferometer is collected by a photodiode with a dynamic range of $5 \mathrm{MHz}$. The oscillator of a lock-in amplifier actuates the PZT and the same lock-in amplifier demodulates the response of the photodiode. Sample and microscope are placed inside a vacuum can, which in turn is mounted inside a liquid helium bath cryostat.

Figure 2(a) shows the measured displacement of a nanowire for various driving amplitudes. As the driving amplitude is increased, the resonance becomes broader and assumes a characteristic shark-fin shape when entering the nonlinear regime, where the frequency associated with maximum displacement increases and moves away from the resonator eigenfrequency $f_{0}$. Such behavior is typical for a Duffing oscillator and can be described by the Duffing equation of motion (including nonlinear damping)

$$
\ddot{x}(t)+\mu \dot{x}(t)+\eta \dot{x}(t) x^{2}(t)+f_{0}{ }^{2} x(t)+\alpha x^{3}(t)=F(t) .
$$

Here, $x$ is the displacement, $\mu$ and $\eta$ are the linear and nonlinear damping constants, and $F(t)$ is the time-dependent driving force, here taken to be sinusoidal. The coefficient $\alpha$ parametrizes the strength of the cubic Duffing nonlinearity. When $\alpha$ is positive, as it is in our case, the Duffing nonlinearity increases the effective spring constant with increasing driving amplitude, thus stiffening the motion. The observed lineshape at higher driving amplitudes is a consequence of Eq. (1) having two stable solutions within a certain frequency range. This bistability leads to the switching
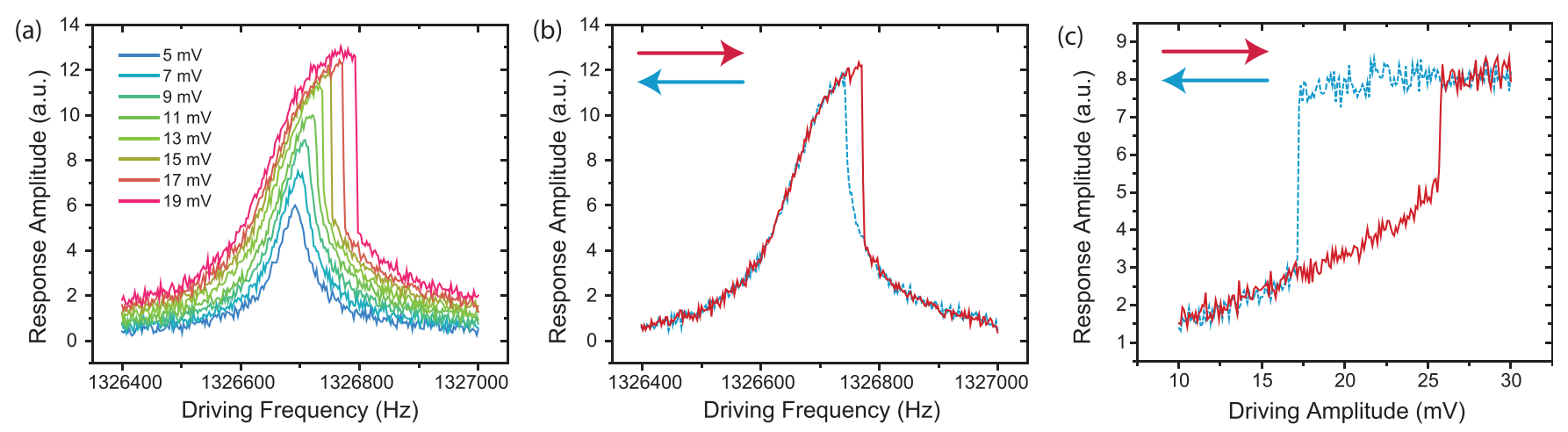

FIG. 2. (a) Response amplitude as a function of driving frequency, for various driving amplitudes. Note that the slight depression around the maximum response for the highest driving amplitude is caused by the limited linear range of the interferometer. (b) Response amplitude as a function of driving frequency (at a driving amplitude of $17 \mathrm{mV}$ ), for two sweep directions (as indicated by arrows). (c) Response amplitude as a function of driving amplitude (at a driving frequency of $1326770 \mathrm{~Hz}$ ), for two sweep directions. 
phenomena seen at the right flank of the response peak (Fig. 2(a)). Which of the two solutions is realized, is determined by the initial conditions, and mechanical hysteresis can be observed when adiabatically sweeping the driving frequency or driving amplitude up and down (Figures 2(b) and 2(c)). The strength of the nonlinearity $\alpha$ can be estimated from the shift of the frequency $f_{\text {max }}$ at which the maximum response amplitude occurs, using the relation: ${ }^{21} \alpha=\frac{32}{3} \pi^{2} f_{0}\left(f_{\text {max }}-f_{0}\right) / x^{2}$. Our interferometer becomes nonlinear for larger driving amplitudes (see Fig. 2(a)), since then the displacement becomes comparable to the width of the interferometer fringes. We use this to infer $^{29}$ a value for the displacement $x$ of $\sim 250 \mathrm{~nm}$, for a driving amplitude of $19 \mathrm{mV}$ (Fig. 2(a)). We can then estimate $\alpha$ to be of order $10^{23} \mathrm{~m}^{-2} \mathrm{~s}^{-2}$ for this nanowire.

Nonlinear damping could arise in the presence of a Duffing nonlinearity and linear damping. ${ }^{9}$ We briefly discuss this and show that in our case nonlinear damping does not play a significant role. The nonlinear damping term has the effect of decreasing the shift of the frequency of maximum response amplitude due to the Duffing nonlinearity, as well as decreasing the size of the hysteresis loop. The coefficient for nonlinear damping $\eta$ can be estimated from the critical frequency, which is the frequency at which the system, with sufficient driving strength, turns from being monostable to being bistable. This critical frequency has a minimum when nonlinear damping is absent, which corresponds to $\sqrt{3} f_{0} / 2 Q .{ }^{17}$ This yields $\sim 52 \mathrm{~Hz}$ in our case, agreeing very well to the value of $49 \mathrm{~Hz}$ determined from the measurements, indicating that here $\eta$ is negligible.

Next, we show that the nonlinearity can be used to turn a nanowire into a mechanical mixer. ${ }^{19,20}$ Upon excitation with two driving frequencies, $F(t)=F_{1} \cos \left(f_{1} t+\phi_{1}\right)+F_{2}$ $\cos \left(f_{2} t+\phi_{2}\right)$, the response shows sidebands additional to the motion at the driving frequencies, as shown in Figure 3(a). We observe up to twelve such sidebands, spaced around the driving frequencies with splittings equal to the detuning between the two driving frequencies, $\Delta f=f_{2}-f_{1}$. Note that the results shown in Fig. 3 were obtained by measuring on a second nanowire. Measurements on both nanowires were very similar, but the signal to noise ratio is varied.

This response can be understood from Eq. (1) by taking the cubic term to be a perturbation to the driving force and solving the equation iteratively. ${ }^{21,30}$ One then obtains new terms in the response at the intermodulation frequencies $f_{1}-n \Delta f$ and $f_{2}+n \Delta f$ (where $n$ is an integer) for each iteration. The amplitudes of these new intermodulation terms have coefficients proportional to $\sum_{n}\left(f_{0}^{2}-f_{1}^{2}\right)^{-k}\left(f_{0}^{2}-f_{2}^{2}\right)^{-l}$, with $k$ and $l$ positive integers and $k+l=n$. Hence, intermodulation terms are smaller for driving frequencies that are more detuned from resonance. Since the mixing occurs due to the cubic term in Eq. (1), for the intermodulation terms to be present, at least one of the driving amplitudes needs to be large enough to have an appreciable nonlinear response.

It is evident from Fig. 3 that the energy that is injected into the nanowire oscillator by the driving is distributed among the various intermodulation terms. This redistribution also occurs when one drive (signal) is much smaller than the other (pump), in which case amplification of the signal can take place. ${ }^{17}$ The signal here is formed by a driving voltage supplied to the PZT, but it could be any force driving the nanowire with a frequency close to the resonance. Fig. 3(b) shows the spectral response of the nanowire motion with the signal drive always on, but with the pump excitation off in one case and on in the other. It is clear that amplification of the signal takes place when the pump excitation is switched on in the form of an increase in amplitude of the response at the signal frequency. Additionally, the appearance of the intermodulation terms, which is conditional on the presence of a signal, provides extra amplification. The total gain can be defined to be the ratio between the summed response amplitudes of all peaks present with pump drive, excluding the peak at the pump frequency itself, and the response amplitude with no pump drive. We observe a maximum gain of $26 \mathrm{~dB}$. Although higher gain might be expected ${ }^{17}$ in the limit of vanishing detuning between pump and signal frequencies, detection of the signal then becomes impractical. The observation of mechanical frequency mixing is furthermore limited by the onset of nonlinearity of the detection interferometer for high displacement amplitudes. If the interferometer response becomes significantly nonlinear, multiple frequencies in the mechanical response can also lead to new frequency components in the detected signal. However, we already observe intermodulation frequency components for small excitation amplitudes, large enough to reach the
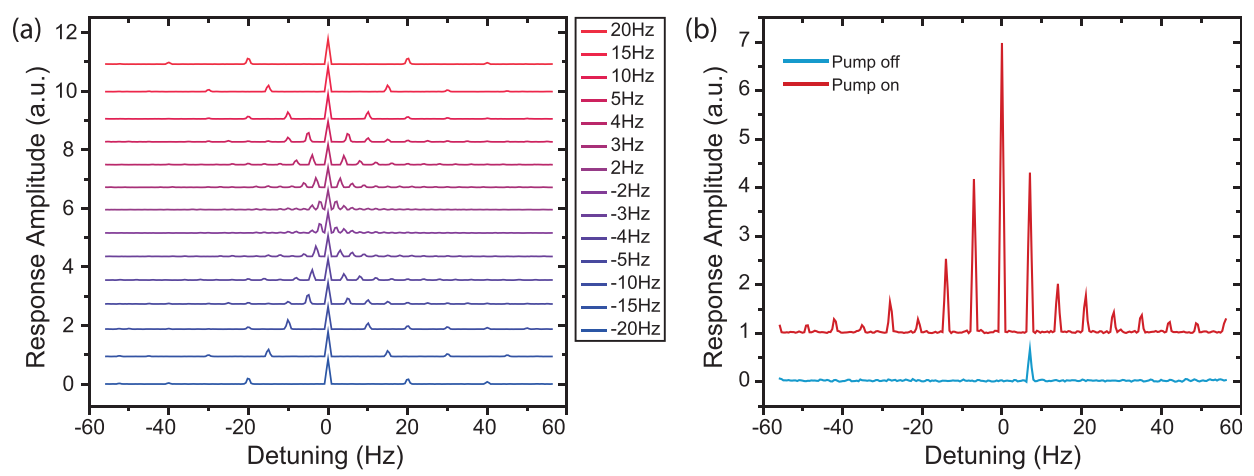

FIG. 3. (a) Spectral response amplitudes of the nanowire motion upon application of two driving frequencies, for various values of the detuning of the signal frequency from the pump frequency. The curves have been offset for clarity. The spectral response is given as a function of the detuning from the pump frequency, which is $1287890 \mathrm{~Hz}$. The pump and signal amplitudes are $250 \mathrm{mV}$ and $50 \mathrm{mV}$, respectively. The first mechanical mode of this nanowire has a resonant frequency of this nanowire at $1287780 \mathrm{~Hz}$. (b) Spectral response with pump excitation (1287913 Hz, $250 \mathrm{mV}$ ) off (lower curve) and on (upper curve) for a signal detuned from the pump frequency by $7 \mathrm{~Hz}$ and with amplitude $35 \mathrm{mV}$. The curves have been offset for clarity. 
mechanical nonlinear regime, but small enough to generate mechanical displacements of up to only $10 \mathrm{~nm}$. For these displacements, the second- and third-order terms in the optical response are smaller than $2 \%$ and $0.02 \%$ of the first-order term, respectively. The amplitude of any optically generated sidebands is therefore very small, indicating the mechanical origin of the observed sidebands.

In summary, we have observed and characterized nonlinear motion of as-grown GaAs nanowires. The nonlinearity is already observable for modest driving amplitudes. Furthermore, we have demonstrated that this nonlinearity allows for mechanical mixing of two excitations and amplification of a signal excitation through this mixing. This amplification could be utilized in several scanning probe techniques. For example, in the case where these nanowires act as mechanical force transducers, the observed gain of $26 \mathrm{~dB}$ could make force sensitivities of $\sim 100 \mathrm{zN} / \sqrt{\mathrm{Hz}}$ in a narrow bandwidth feasible. These results indicate that although nonlinear motion can be non-negligible for nanowires, the nonlinearity can also be turned into an advantage using simple measurement schemes. The nonlinearity could, in addition, lead to coupling of different flexural modes. Such nonlinear mode coupling could have several applications, including tuning the resonance frequency ${ }^{31}$ and quality factor $^{32}$ of one mode through driving of the other mode, and implementing quantum non-demolition measurements of mechanical excitation. ${ }^{33}$

We thank Pengfei Wang, Jiangfeng Du, and Sascha Martin for technical support. This work was supported by an ERC Grant (NWscan, Grant No. 334767), the Swiss Nanoscience Institute (Project P1207), QSIT, and the Sino Swiss Science and Technology Cooperation (Project IZLCZ2 1388904).

${ }^{1}$ M. Poggio, Nat. Nanotechnol. 8, 482-483 (2013).

${ }^{2}$ J. M. Nichol, E. R. Hemesath, L. J. Lauhon, and R. Budakian, Appl. Phys. Lett. 93, 193110 (2008).

${ }^{3}$ J. M. Nichol, T. R. Naibert, E. R. Hemesath, L. J. Lauhon, and R. Budakian, Phys. Rev. X 3, 031016 (2013).

${ }^{4}$ X. L. Feng, R. He, P. Yang, and M. L. Roukes, Nano Lett. 7, 1953-1959 (2007).

${ }^{5}$ I. Yeo, P.-L. de Assis, A. Gloppe, E. Dupont-Ferrier, P. Verlot, N. S. Malik, E. Dupuy, J. Claudon, J.-M. Gérard, A. Auffèves, G. Nogues, S. Seidelin, J.-Ph. Poizat, O. Arcizet, and M. Richard, Nat. Nanotechnol. 9, 106-110 (2014).
${ }^{6}$ M. Montinaro, G. Wüst, M. Munsch, Y. Fontana, E. Russo-Averchi, M. Heiss, A. Fontcuberta i Morral, R. J. Warburton, and M. Poggio, Nano Lett. 14(8), 4454-4460 (2014).

${ }^{7}$ L. G. Villanueva, R. B. Karabalin, M. H. Matheny, D. Chi, J. E. Sader, and M. L. Roukes, Phys. Rev. B 87, 024304 (2013).

${ }^{8}$ R. Lifshitz and M. C. Cross, Nonlinear Dynamics of Nanomechanical and Micromechanical Resonators, Reviews of Nonlinear Dynamics and Complexity (Wiley-VCH, Weinheim, 2008), Vol. 1, pp. 1-52.

${ }^{9}$ A. Eichler, J. Moser, J. Chaste, M. Zdrojek, I. Wilson-Rae, and A. Bachtold, Nat. Nanotechnol. 6, 339-342 (2011).

${ }^{10}$ S. Zaitsev, O. Shtempluck, E. Buks, and O. Gottlieb, Nonlinear Dyn. 67, 859-883 (2012)

${ }^{11}$ F. C. Moon and S. W. Shaw, Int. J. Non-Linear Mech. 18, 465 (1983).

${ }^{12}$ M. Tabaddor, Int. J. Solids Struct. 37, 4915 (2000).

${ }^{13}$ D. Antonio, D. H. Zanette, and D. López, Nat. Commun. 3, 806 (2012).

${ }^{14}$ H. W. Ch. Postma, I. Kozinsky, A. Husain, and M. L. Roukesa, Appl. Phys. Lett. 86, 223105 (2005).

${ }^{15}$ A. Husain, J. Hone, H. W. Ch. Postma, X. M. H. Huang, T. Drake, M. Barbic, A. Scherer, and M. L. Roukes, Appl. Phys. Lett. 83, 1240 (2003).

${ }^{16}$ J. M. Nichol, E. R. Hemesath, L. J. Lauhon, and R. Budakian, Appl. Phys. Lett. 95, 123116 (2009).

${ }^{17}$ R. Almog, S. Zaitsev, O. Shtempluck, and E. Buks, Appl. Phys. Lett. 88, 213509 (2006).

${ }^{18}$ R. B. Karabalin, R. Lifshitz, M. C. Cross, M. H. Matheny, S. C. Masmanidis, and M. L. Roukes, Phys. Rev. Lett. 106, 094102 (2011).

${ }^{19}$ R. Almog, S. Zaitsev, O. Shtempluck, and E. Buks, Phys. Rev. Lett. 98, 078103 (2007).

${ }^{20}$ A. Erbe, H. Krömmer, A. Kraus, R. H. Blick, G. Corso, and K. Richter, Appl. Phys. Lett. 77, 3102 (2000).

${ }^{21}$ A. H. Nayfeh and D. T. Mook, Nonlinear Oscillations (Wiley, New York, 1979).

${ }^{22}$ V. Barger and M. Olsson, Classical Mechanics: A Modern Perspective, 2nd ed. (McGraw-Hill, Inc., New York, 1995).

${ }^{23}$ X. Y. Lü, J. Q. Liao, L. Tian, and F. Nori, e-print arXiv:1403.0049.

${ }^{24}$ C. Colombo, D. Spirkoska, M. Frimmer, G. Abstreiter, and A. Fontcuberta i Morral, Phys. Rev. B 77, 155326 (2008).

${ }^{25}$ M. Heiss, Y. Fontana, A. Gustafsson, G. Wüst, C. Magen, D. D. ORegan, J. W. Luo, B. Ketterer, S. Conesa-Boj, A. V. Kuhlmann, J. Houel, E. Russo-Averchi, J. R. Morante, M. Cantoni, N. Marzari, J. Arbiol, A. Zunger, R. J. Warburton, and A. Fontcuberta i Morral, Nat. Mater. 12, 439-444 (2013).

${ }^{26}$ K. Y. Yasumura, T. D. Stowe, E. M. Chow, T. Pfafman, T. W. Kenny, B. C. Stipe, and D. Rugar, J. Microelectromech. Syst. 9, 117-125 (2000).

${ }^{27}$ J. Yang, T. Ono, and M. Esashi, J. Microelectromech. Syst. 11, 775-783 (2002).

${ }^{28}$ A. Högele, S. Seidl, M. Kroner, K. Karrai, C. Schulhauser, O. Sqalli, J. Scrimgeour, and R. J. Warburton, Rev. Sci. Instrum. 79, 023709 (2008).

${ }^{29}$ M. Dobosz, T. Usuda, and T. Kurosawa, Meas. Sci. Technol. 9, 232 (1998).

${ }^{30}$ C. Hutter, D. Platz, E. A. Tholén, T. H. Hansson, and D. B. Haviland, Phys. Rev. Lett. 104, 050801 (2010).

${ }^{31}$ R. B. Karabalin, M. C. Cross, and M. L. Roukes, Phys. Rev. B 79, 165309 (2009).

${ }^{32}$ W. J. Venstra, H. J. R. Westra, and H. S. J. van der Zant, Appl. Phys. Lett. 99, 151904 (2011).

${ }^{33}$ D. H. Santamore, A. C. Doherty, and M. C. Cross, Phys. Rev. B 70, 144301 (2004). 